\title{
Atendimento Odontológico à Gestante - Parte 1: Alterações Sistêmicas
}

\author{
SILVA, Francisco Wanderley Garcia de Paula e * $_{*}^{*}$ \\ STUANI, Adriana Sasso \\ QUEIROZ, Alexandra Mussolino de
}

\begin{abstract}
RESUMO
Diversas alterações fisiológicas acontecem durante a gestação e, embora visem proteger o feto, podem debilitar as mulheres grávidas, tornando-as mais suscetíveis a distúrbios sistêmicos. O conhecimento da história médica da paciente, por meio do preenchimento de uma ficha de anamnese detalhada, é fundamental para o estabelecimento de um plano de tratamento seguro. Assim, o objetivo deste trabalho é relatar as principais alterações sistêmicas relacionadas à gestação, possibilitando ao cirurgião dentista diferenciar as alterações fisiológicas daquelas consideradas patológicas e prestar atendimento odontológico individualizado, conhecendo as limitações de cada paciente.
\end{abstract}

PALAVRAS-CHAVES:

Alterações sistêmicas. Gestação. Atendimento odontológico.

\section{INTRODUCÃO}

Diversas alterações imunológicas, bioquímicas e hemodinâmicas acontecem durante a gestação e estão relacionadas principalmente ao aumento na secreção de hormônios sexuais e ao crescimento e desenvolvimento do bebê (WEISS, 2000).

Essas mudanças fisiológicas, embora visem proteger o feto, podem debilitar as mulheres grávidas, tornando-as mais suscetíveis a distúrbios sistêmicos (SUSANTI et al., 2001). Dentre as principais alterações que podem ocorrer durante a gestação estão aquelas relacionadas aos sistemas cardiovascular, respiratório, renal, gastrointestinal e endócrino (SURESH; RADFAR, 2004).

Em decorrência das alterações próprias da gestação, alguns cuidados passam a ser necessários durante o atendimento odontológico. Porém, na presença de distúrbios pré-existentes, a gravidez pode se tornar um período complicado tanto para a gestante quanto para o bebê.

Independentemente da presença de distúrbios durante a gestação, o conhecimento da história médica da paciente, por meio do preenchimento de uma ficha de anamnese detalhada, é fundamental para o estabelecimento de um plano de tratamento seguro. (JAMES; NELSON-

1. Aluno do Curso de Pós-graduação em Odontopediatria da Faculdade de Odontologia de Ribeirão Preto/USP 2. Professora Assistente do Departamento de Clínica Infantil, Odontologia Preventiva e Social da Faculdade de Odontologia de (bpm) e pode sofrer uma elevação da ordem de 10 a $15 \mathrm{bpm}$ a partir da $14^{\mathrm{a}}$ até a $30^{\mathrm{a}}$ semana de gestação (FERRERO et al., 2004; ABBAS et al., 2005). Essas mudanças acometem $90 \%$ das mulheres grávidas (THORNBURG et al., 2000) e acontecem para atender às demandas do feto (GOWDA et al., 2003; ABBAS et al., 2005).

Algumas dessas alterações podem agravar um problema cardíaco pré-existente e levar a morbidade e mortalidade (FERRERO et al., 2004). Por esse motivo, é fundamental que o cirurgião dentista conheça a história médica da paciente e, na presença de arritmias, com sintomas clássicos como palpitação, fadiga, dispnéia, tonturas, dor opressiva no peito e visão escurecida, que indicam comprometimento cardíaco grave (ABBAS et al., 2005), solicite avaliação médica da

Com relação à pressão arterial, esta é caracterizada por uma queda no primeiro trimestre da gestação, causada por uma vasodilatação pela ação de mediadores locais como a prostaciclina e o óxido nítrico. Esta redução de $10 \mathrm{mmHg}$ na pressão arterial acontece primeiramente na pressão diastólica, na $13^{\mathrm{a}}-20^{\mathrm{a}}$ semana da gestação. A pressão continua a diminuir até a $22^{\text {a }}-24^{a}$ semana, quando é estabilizada e inicia o aumento gradual paciente. cia de ỡ mente é de 72 batimentos por minuto 
até o parto (SIBAI, 1996). Porém, por volta da $28^{a}$ semana, as pacientes estão predispostas a hipotensão devido a compressão da veia cava (MILLS; MOSES, 2002). Essa condição é conhecida como síndrome da hipotensão postural, acomete $8 \%$ das gestantes é caracterizada por hipotensão, náusea, tontura e fraqueza (LANNI et al., 2002).

Durante a gravidez podem existir 3 tipos principais de desordens na pressão arterial, que são: a hipertensão crônica, a hipertensão gestacional e a pré-eclâmpsia. A hipertensão é denominada crônica quando existe previamente à gravidez, a gestacional é caracterizada pelo aumento da pressão arterial em mulheres normotensas, geralmente no segundo trimestre da gravidez e a pré-eclâmpsia é caracterizada por um quadro de hipertensão, edema e proteinúria que geralmente leva ao parto prematuro de bebês de baixo peso (JAMES; NELSON-PIERCE, 2004).

Caracteriza-se hipertensão na gravidez quando a pressão arterial sistólica (PAS) estiver $>140 \mathrm{mmHg}$ e a diastólica (PAD) $>90 \mathrm{mmHg}$, em duas tomadas, em repouso, com intervalo de $4 \mathrm{~h}$ entre as medições, ou quando houver aumento $>30 \mathrm{mmHg}$ na PAS e/ou aumento $>15 \mathrm{mmHg}$ na $\mathrm{PAD}$, em relação aos valores considerados normais previamente à gestação (JAMES; NELSON-PIERCE, 2004).

\section{Alterações hematológicas}

$\mathrm{O}$ aumento do volume sangüíneo em função da maior quantidade de líquidos no organismo, bem como modificações associadas à coagulação são processos adaptativos da mulher à gestação (FERRERO et al., 2004).

O maior volume sangüíneo acontece devido ao aumento do volume do plasma de um baseline de 40 para $79 \mathrm{~mL} / \mathrm{kg}$ por volta da $32^{\mathrm{a}}$ semana de gestação. $O$ volume de células vermelhas também aumenta de 25 para $30 \mathrm{~mL} / \mathrm{kg}$, porém numa velocidade mais lenta, causando a chamada anemia relativa da gestação (ABBAS et al., 2005).

O aumento na concentração de fatores de coagulação, diminuição da concentração de alguns anticoagulantes naturais e diminuição da atividade fibrinolítica, mantém a função plaquetária durante a gestação e são responsáveis pela hemostasia pós-parto (BREMME, 2003). Estas alterações acontecem, provavelmente, em decorrência de mudanças hormonais e protegem a mulher de hemorragia durante o parto, entretanto predispõem ao tromboembolismo (SATTAR et al., 1999).

\section{Alterações respiratórias}

São caracterizadas por um aumento na freqüência respiratória e no consumo de oxigênio, uma vez que a mulher necessita de mais oxigênio durante a gestação, tanto para si mesma como para o bebê. Nesse período, acontece também uma queda no volume respiratório residual em cerca de $20 \%$ pela elevação do diafragma para acomodar o útero em crescimento (WEINBERGER et al., 1980; SUSANTI et al., 2001; EDWARDS et al., 2005).

A mucosa das vias aéreas pode apresentar hiperemia, hipersecreção e edema, principalmente no $3^{\circ}$ trimestre, o que pode predispor a gestante a eventos obstrutivos das vias aéreas superiores. Podem surgir, ainda, desordens respiratórias do sono, como o ronco, que reflete um aumento na incidência da síndrome da apnéia obstrutiva nesse período (SANTIAGO et al., 2001).

\section{Alterações metabólicas}

As alterações metabólicas acontecem principalmente pelo aumento da taxa de excreção renal e em função das alterações hepáticas (FERRERO et al., 2004). Pode ocorrer ainda fadiga no metabolismo de carboidratos, exigindo aumento nos níveis de insulina, surgindo assim, o diabetes gestacional (FORSBACH-SANCHEZ et al., 2005).

O diabetes melittus gestacional é definido como uma intolerância à glicose detectada durante a gestação, cujos fatores de risco associados são a obesidade, a história familiar, a idade precoce ou avançada da gestante e a história prévia de diabetes (The Expert Committee on the Diagnosis and Classification of Diabetes Mellitus, 2003).

Para a detecção clínica do diabetes mellitus, dois procedimentos têm sido propostos. O procedimento da ADA (American Diabetes Association) consiste em um teste imediato de glicemia após ingestão de $50 \mathrm{~g}$ de glicose, sendo o nível glicêmico considerado normal se menor que $140 \mathrm{mg} / \mathrm{dL}$. Se este valor estiver aumentado, um novo teste deve ser realizado com 100g de glicose, após 1, 2 e 3 horas. É classificado como diabetes se dois valores forem coincidentes com o de $140 \mathrm{mg} / \mathrm{dL}$ ou maiores. O método da OMS (Organização Mundial da Saúde) é mais simples e consiste na administração de $75 \mathrm{~g}$ de glicose e medição do nível de glicemia 2 horas depois, sendo o valor considerado normal se menor que $140 \mathrm{mg} /$ dL (FORSBACH-SANCHEZ et al., 2005).

Se a condição de diabetes gestacional for conhecida, é importante observar se o controle dietético foi estabelecido, se este está sendo seguido, devendo-se ainda interrogar a paciente quanto à terapia com insulina (FORSBACH-SANCHEZ et al., 2005).

$O$ aumento na demanda de energia para o crescimento do feto e da placenta afeta o metabolismo de alguns nutrientes, causando muitas vezes a deficiência de ferro e ácido fólico. Como o íon ferro é importante para a eritropoese e o acido fólico para a síntese de aminoácidos e ácidos nucléicos, suplementos dessas substâncias devem ser administrados durante a gestação (CASANUEVA et al., 2003).

\section{Alterações gastrointestinais}

Durante a gestação ocorre a diminuição da motilidade intestinal. Estudos recentes têm mostrado que esta mudança está relacionada aos níveis aumentados de hormônios sexuais e não ao aumento do volume do útero como se acreditava antigamente (BARON et al., 1993). A secreção gástrica também está diminuída neste período, o que pode afetar a absorção gastrointestinal (FERRERO et al., 2004).

A êmese, ou seja, reflexo de náusea e vômito matutinos, acontece em $1 \mathrm{em}$ cada 200 gestantes (BARON et al., 1993). É importante salientar que embora seja considerado normal durante a gravidez, em algumas situações mais graves, como a hiperêmese, pode levar a desidratação e perda de peso (QUINLA; HILL, 2003). A etiologia desse problema ainda é desconhecida, mas tem sido associada à elevação dos níveis de gonadotrofina coriônica e à hipoglicemia (MASSON et al., 1985; BARON et al., 1993). O tratamento dessa enfermidade é de ordem médica e, dependendo da severidade dos sintomas, varia de mudança de hábitos alimentares à utilização de medicamentos antieméticos, hospitalização e necessidade de alimentação parenteral (QUINLA; HILL, 2003).

\section{Alterações Hormonais}

A placenta é um órgão altamente vascularizado que se desenvolve nas primeiras semanas da gestação para fornecer oxigênio e nutrientes ao feto e remover seus produtos de excreção (GUYTON, 1991). As alterações hormonais intensas, durante a gestação, como o aumento da elaboração de gonadotrofina coriônica, estrogênios, progesterona e somatomamotropina (hormônio lactogênio placentário) estão relacionados a esse órgão. 
A gonadotrofina coriônica é um hormônio importante no início da gestação, cuja função é manter o corpo lúteo até que a placenta esteja apta a produzir estrógeno e progesterona.

A progesterona é responsável pela implantação do embrião, estimula o desenvolvimento das células deciduais que mantêm a nutrição do feto e das glândulas mamárias para produção de leite. No final da gestação, está relacionada à indução do parto.

O estrógeno estimula o crescimento uterino, o aumento de tamanho dos seios e o relaxamento dos ligamentos pélvicos. Durante a gravidez, os níveis de estrógeno são 100 vezes maiores que os níveis basais, sendo produzidos principalmente o estradiol, o estriol e a estrona.

A somatomamotropina é semelhante ao hormônio do crescimento e estimula o desenvolvimento do feto. Na mãe diminui a sensibilidade à insulina, aumentando os níveis plasmáticos de glicose para o feto (WEISS, 2000).

Há também o aumento da secreção hipofisária (tireotropina e prolactina), secreção adrenocortical de glicocorticóides, secreção de hormônios tireoideanos (tiroxina), secreção das glândulas paratireóides (GLINOER et al., 1990; TRAINER, 2002). Essa maior secreção paratireoidea é responsável pelo aumento dos níveis séricos de vitamina $D$, principalmente se a mãe apresentar deficiência de cálcio (RASMUSSEN et al., 1990). Assim, acontece a reabsorção de cálcio dos ossos longos da mãe mantendo normal a concentração de íons cálcio no sangue para a ossificação fetal (GUYTON, 1991).

\section{Alterações bucais}

Durante a gestação, a cavidade bucal sofre algumas mudanças e uma maior prevalência de alterações têm sido relatada. A principal delas é a doença cárie, que acomete $99,38 \%$ das gestantes (MAUSEBERG et al., 1991; RUIZ et al., 2002). Entretanto, essa patologia não é predisposta pela gestação, mais sim dependente das alterações alimentares, como o maior consumo de açúcares e a negligência dos hábitos de higiene bucal (CASTELLANOS et al., 2002; GAJENDRA; KUMAR, 2004). As alterações salivares, como a mudança na composição e a menor taxa de secreção, também podem estar relacionadas ao risco a cárie aumentado nesse período. As mudanças na composição da saliva, que incluem diminuição de sódio, aumento nos níveis de potássio, proteínas e estrógeno (SALVOLINI et al., 1998) levam à queda de
pH, reduzindo, portanto, a sua capacidade tampão (SURESH; RADFAR, 2004).

A gengivite é uma alteração comum durante a gravidez e alguns autores consideram normal o sangramento nessas pacientes. A prevalência da gengivite varia de 50 a 98,25\% (CASTELLANOS et al., 2002; RUIZ et al., 2002) e está relacionada ao aumento dos níveis hormonais, que parecem exacerbar a resposta gengival ao biofilme dental (AMERICAN DENTAL ASSOCIATION, 1995; GAJENDRA; KUMAR, 2004.) Ainda, a progesterona e o estrógeno, por exemplo, aumentam a vascularização, contribuindo assim para uma maior intensidade das respostas inflamatórias (DIAZGUZMAN; SUAREZ, 2004).

A progesterona causa o aumento do exsudato, afeta a integridade das células endoteliais e influencia na biosíntese de prostaglandina na gengiva (DIAZGUZMAN; SUAREZ, 2004). Por esse motivo, a gengiva se torna inflamada, edematosa, sensível e com tendência ao sangramento (GAJENDRA; KUMAR, 2004). Receptores específicos para estrógeno e progesterona foram demonstrados no tecido gengival (VITTEK et al., 1982), sugerindo que esse tecido pode funcionar como um órgão-alvo desses hormônios sexuais. Como as alterações gengivais são bem conhecidas durante a gestação, sabe-se que a gengivite gravídica é uma condição reversível, localizada ou generalizada e mais comum nos dentes anteriores (HUNTER; HUNTER, 1997). Muramatsu et al. (1994) observaram que a gengivite inicia-se no segundo mês de gestação e aumenta acentuadamente até atingir o pico no oitavo mês, havendo posteriormente uma regressão.

Em resumo, a gengivite é um fenômeno comum em mulheres grávidas e provavelmente está relacionada à presença de biofilme em um hospedeiro fisiologicamente alterado em função da gravidez. Entretanto, a manutenção da higiene bucal pode ajudar na prevenção ou redução da severidade dessas alterações inflamatórias mediadas pelo estrógeno e progesterona.

Com relação à periodontite, está demonstrado que não é causada pela gravidez, entretanto, a doença periodontal pré-existente pode ser exacerbada pelos níveis hormonais alterados nesse período (TILAKARATNE et al., 2000). A relevância clínica disso é que estudos têm mostrado que essa infecção contribui para o nascimento de bebês de baixo peso (OFFENBACHER et al., 1996;
OFFENBACHER; SLADE, 1998; LOPEZ et al., 2002). As bactérias gram negativas responsáveis por essa condição são capazes de produzir uma variedade de mediadores químicos da inflamação, como as prostaglandinas, interleucinas e fator de necrose tumoral que podem afetar diretamente o hospedeiro (OFFENBACHER et al., 1996). Offenbacher et al. (1996) e Offenbacher e Slade (1998) observaram que mulheres com doença periodontal severa podem apresentar níveis elevados desses mediadores que estão relacionados à indução do parto e nascimento de bebês de baixo peso. Estima-se que mais de $18 \%$ dos pré-termos possam ser resultado de doenças periodontais não tratadas.

$O$ granuloma piogênico acomete de 1 a 5\% das mulheres grávidas (VAZQUEZ et al., 1994) e acontece principalmente devido à angiogênese aumentada pelos níveis plasmáticos de estrógeno elevados, associado à irritação local como trauma, biofilme e calculo dental. É uma lesão proliferativa não neoplásica, caracterizada como um processo inflamatório cuja localização mais comum é na gengiva na região anterior da maxila (RIVERO; ARAÚJO, 1998). Clinicamente apresenta-se como uma massa nodular de crescimento lento, avermelhada, mole à palpação e com tendência à hemorragia espontânea. $\mathrm{O}$ aspecto histológico da lesão mostra massas lobulares de tecido de granulação, proliferação de fibroblastos e células endoteliais e formação de numerosos vasos sangüíneos (AL-KHATEEB; ABABNEB, 2003). O tratamento consiste na remoção cirúrgica e eliminação de qualquer fator local que possa atuar como irritante e acarretar a recidiva da lesão, embora seja relatado que possam regredir espontaneamente após o nascimento do bebê (DIAZ-GUZMAN; SUAREZ, 2004).

Quanto ao risco de erosão dental, em função dos episódios de vômito, este tem sido relatado na literatura, mas ainda não existem estudos clínicos que comprovem esses achados (MAUSEBERG et al., 1991; RUIZ et al., 2002; GAJENDRA; KUMAR, 2004). Hunter e Hunter (1997) relataram que as superfícies palatinas dos dentes anteriores superiores são as mais afetadas. Assim, é importante recomendarmos às nossas pacientes gestantes que escovem os dentes ou façam uso de um enxagüatório bucal fluoretado imediatamente após cada episódio de vômito, para evitar a erosão do esmalte dental (MILLS; MOSES, 2002; GAJENDRA; KUMAR, 2004). 


\section{CONCLUSÃO}

Alterações cardiovasculares, hematológicas, respiratórias, renais, gastrointestinais e endócrinas podem se manifestar durante a gestação. O conhecimento dessas alterações fisiológicas, por parte do cirurgião dentista, associado à coleta de dados sobre a história médica anterior e atual da gestante, por meio de uma anamnese bem realizada, é de fundamental importância para que se estabeleça um plano de tratamento odontológico seguro e individualizado para a paciente.

\section{ABSTRACT}

Several physiologic alterations can occur during pregnancy which, if even they happen to protect the fetus, may debilitate the pregnant woman that becomes susceptible to systemic disorders. Knowledge of patient medical history by a full detailed anamnesis form is important to establish the best ways of a safe treatment. Thus, the aim of this study is to relate the main systemic alterations during pregnancy, making possible to the dentist the differentiation between physiologic alterations and others considered pathologic, and give to the pregnant woman an individualized dental assistance knowing the limitations of each patient.

\section{KEY WORDS}

Pregnancy. Systemic alterations. Dental assistance.

\section{REFERÊNCIAS}

ABBAS, A.E.; LESTERA, S.J.; CONNOLLY, H. Pregnancy and the Cardiovascular System. Int. J. Cardiol., Amsterdam, v.98, p.179-189, 2005.

AL-KHATEEB, T.; ABABNEH, K. Oral Pyogenic Granuloma in Jordanians: A Retrospective Analysis of 108 Cases. J. Oral Maxillofac. Surg., Philadelphia, v.61, p.1285-1288, 2003.

AMERICAN DENTAL ASSOCIATION COUNCIL ON ACCESS, PREVENTION, AND INTERPROFESSIONAL RELATIONS. ADA Oral Health Care Series: Women's Oral Health Issues. Chicago: American Dental Association, 1995.

BARON, T.H.; RAMIREZ, B.; RICHTER, J.E. Gastrointestinal Motility Disorders During Pregnancy. Ann. Intern. Med., Philadelphia, v.118, p.366-375, 1993.
BREMME, K.A. Haemostatic Changes in Pregnancy. Best Pract. Res. Clin. Haematol., London, v.16, no.2, p.153-168, 2003.

CASANUEVA, E. et al. Iron and Folate Status Before Pregnancy and Anemia During Pregnancy. Ann. Nutr. Metab., Basel, v.47, p.60-63, 2003.

CASTELLANOS, J.L.; DÍAZ, G.L.; GAY, O. Medicina en Odontología: manejo de pacientes con enfermedades sistémicas. México: Manual Moderno, 2002.

DIAZ-GUZMAN, L.M.; CASTELLANOS-SUAREZ, J.L. Lesiones de la mucosa bucal y comportamiento de la enfermedad periodontal en embarazadas. Med. Oral Patol. Oral Cir. Bucal, Valencia, v.9, p.430-437, 2004.

EDWARDS, N. et al. Sleep Disordered Breathing and Pregnancy. Thorax, London, v.57, p.555-558, 2002.

THE EXPERT COMMITTEE ON THE DIAGNOSIS AND CLASSIFICATION OF DIABETES MELLITUS. Report of the Expert Committee on the Diagnosis and Classification of Diabetes Mellitus. Diabetes Care, New York, v.26, p.5-20, 2003.

FERRERO, S.; COLOMBO, B.M.; RAGNI, N. Maternal Arrhythmias During Pregnancy. Arch. Gynecol. Obstet., München, v.269, p.244-253, 2004.

FORSBACH-SANCHEZ, G.; TAMEZ-PEREZ, H.E.; VAZQUEZLARA, J. Diabetes and Pregnancy. Arch. Med. Res., New York, v.36, p.291-299, 2005.

GAJENDRA, S.; KUMAR, J.V. Oral Health and Pregnancy: A Review. N. Y. State Dent. J., New York, p.40$44,2004$.

GLINOER, D. et al. Regulation of Maternal Thyroid During Pregnancy. J. Clin. Endocrinol. Metab., Springfield, v.71, p.276-287, 1990.

GOWDA, R.M.; et al. Cardiac Arrhythmias in Pregnancy: Clinical and Therapeutic Considerations. Int. J. Cardiol., Amsterdam, v.88, p.129-
$133,2003$.

GUYTON, A.C. Textbook of Medical Physiology. 8. ed. Philadelphia: WB Saunders, 1991.

HUNTER, L.; HUNTER, B. Oral and Dental Problems Associated with Pregnancy. London: Macmillan Press, 1997.

JAMES, P.R.; NELSON-PIERCY, C. Management of Hypertension Before, During, and After Pregnancy. Heart, London, v.90, p.1499-1504, 2004.

LANNI, S.M.; TILLINGHAST, J.; SILVER, H. Hemodynamic Changes and Baroreflex Gain in the Supine Hypotensive Syndrome. Am. J. Obstet. Gynec., St. Louis, v.187, p.16361641, 2002.

LOPEZ, N.J.; SMITH, P.C.; GUTIERREZ, J. Periodontal Therapy May Reduce the Risk of Preterm Low Birth Weight in Women with Periodontal Disease: A Randomized Controlled Trial. J. Periodontol., Chicago, v.73, p.911-924, 2001.

MASSON, G.M; ANTHONY, F.; CHAU, E. Serum Chorionic Gonadotrophin (hCG), Schwangerschafts Protein 1 (SP1), Progesterone and Oestradiol Levels in Patients with Nausea and Vomiting in Early Pregnancy. Br. J. Obstet. Gynaecol., Oxford, v.92, p.211-215, 1985.

MAUSEBERG, R. et al. Need for Preventive Dentistry in Adult Women: Investigation in Maternal Convalescent Homes 2. Tooth and Periodontal Findings. Oral Prophylaxe, Frankfurt, v.13, p.76-80, 1991.

MILLS, L.W.; MOSES, D.T. Oral Health During Pregnancy. MCN, New York, v.27, p.275-281, 2002.

MURAMATSU, Y.; TAKAESU, Y. Oral Health Status Related to Subgingival Bacterial Flora and Sex Hormones in Saliva During Pregnancy. Bull. Tokyo Dent. Coll., Tokyo, v.35, no.3, p.139-151, 1994.

OFFENBACHER, S. et al. Periodontal Infection as a Possible Risk Factor for Preterm Low Birthweight. J. Periodontol., Chicago, v.67, no.10, p.1103-1113, 1996. 
OFFENBACHER, S.; SLADE, G. Role of Periodontitis in Systemic Health: Spontaneous Preterm Birth. J. Dent. Educ., Washington, v.62, no.10, p.852-858, 1998.

QUINLA, J.D.; HILL, D.A. Nausea and Vomiting of Pregnancy. Am. Fam. Physician, Kansas City, v.68, no.1, p.121-128, 2003.

RASMUSSEN, N. et al. Serum Ionized Calcium and Intact Parathyroid Hormone Levels During Pregnancy and Postpartum. Br. J. Obstet. Gynaecol., Oxford, v.97, p.857-859, 1990.

RIVERO, E.R.C.; ARAÚJO, L.M.A. Granuloma Piogênico: Uma Análise Clínico-Histopatológica de 147 Casos Bucais. Rev. Fac. Odontol. Univ. Passo Fundo, Passo Fundo, v.3, p.55-61, 1998.

RUIZ, L.G.; GÓMEZ, G.R.; RODRÍGUEZ, G.R. Relación entre la prevalencia de caries dental y embarazo. Rev. Asoc. Dental Mexicana, Ciudad de México, v.59, p.5-9, 2002.

SALVOLINI, E. et al. Biochemical Modifications of Human Whole Saliva Induced by Pregnancy. Br. J. Obstet. Gynaecol., Oxford, v.105, p.656$660,1998$.
SANTIAGO, J.R. et al. Sleep and Sleep Disorders in Pregnancy. Ann. Intern. Med., Philadelphia, v.134, p.396408, 2001.

SATTAR, N. et al. A Longitudinal Study of the Relationships Between Haemostatic, Lipid and Oestradiol Changes During Normal Human Pregnancy. Thromb. Haemost., Stuttgart, v.81, p.71-75, 1999.

SIBAI, B.M. Treatment of Hypertension in Pregnancy. N. Engl. J. Med., Boston, v.335, p.257-265, 1996.

SURESH, L.; RADFAR, L. Pregnancy and Lactation. Oral Surg. Oral Med. Oral Pathol. Oral Radiol. Endod., St. Louis, v.97, p.672-682, 2004.

SUSANTI, I. et al. Respiratory Complications of Pregnancy. Obst. Gynecol. Surv., Baltimore, v.57, p.39-46, 2001.

THORNBURG, K.L. et al. Hemodynamic Changes in Pregnancy. Semin. Perinatol., Philadelphia, v.24, p.11-14, 2000.

TILAKARATNE, A. et al. Periodontal Disease Status During Pregnancy and 3 Months Post-Partum in Rural Population of Sri-Lankan Women. J. Clin. Periodontol., Copenhagen, v.27, p.787-792, 2000 .
TRAINER, P.J. Corticosteroids and Pregnancy. Semin. Reprod. Med., New York, v.20, p.375-380, 2002.

VÁZQUEZ, R.L.; OVALLE, C.J.W. Lesiones bucopatológicas más frecuentes en la mujer embarazada. Rev. Asoc. Dental Mexicana, Ciudad de México, v.51, p.21-28, 1994.

VITTEK, J. et al. Specific Progesterone Receptors in Rabbit Gingiva. J. Periodontal Res., Copenhagen, v.17, no.6, p.657-661, 1982.

WEINBERGER, S.E. et al. Pregnancy and the Lung. Am. Rev. Respir. Dis., Baltimore, v.121, p.559-581, 1980 .

WEISS G. Endocrinology of Parturition. J. Clin. Endocrinol. Metab., Springfield, v.85, p. 4421-4425, 2000.

Endereço para Correspondência: Alexandra Mussolino de Queiroz Departamento de Clínica Infantil, Odontologia Preventiva e Social Faculdade de Odontologia de Ribeirão Preto - Universidade de São Paulo

Avenida do Café, s/n - Bairro Monte Alegre - CEP: 14040-904 - Ribeirão Preto - SP

Telefone: (16) 3602-4116

e-mail: amqueiroz@forp.usp.br 